\title{
Electrosynthesis of Biocompatible Free-Standing PEDOT Thin Films at a Polarised Liquid|Liquid Interface
}

Rob A. Lehane, ${ }^{\mathrm{a}, \mathrm{b}, \dagger}$ Alonso Gamero-Quijano, ${ }^{*, \mathrm{a}, \mathrm{b}, \dagger}$ Sigita Malijauskaite, ${ }^{\mathrm{a}, \mathrm{b}}$ Angelika Holzinger, ${ }^{\mathrm{a}, \mathrm{b}}$ Michelle Conroy, ${ }^{\mathrm{a}, \mathrm{c}}$ Fathima Laffir, ${ }^{\mathrm{a}}$ Amit Kumar, ${ }^{\mathrm{d}}$ Ursel Bangert, ${ }^{\mathrm{a}, \mathrm{c}}$ Kieran McGourty, ${ }^{\mathrm{a}, \mathrm{b}, \mathrm{e}}$ and Micheál D. Scanlon*,a,b,f

a Bernal Institute, University of Limerick (UL), Limerick V94 T9PX, Ireland

${ }^{\mathrm{b}}$ Department of Chemical Sciences, School of Natural Sciences, University of Limerick (UL), Limerick V94 T9PX, Ireland

${ }^{c}$ Department of Physics, School of Natural Sciences, University of Limerick (UL), Limerick V94 T9PX, Ireland

${ }^{\mathrm{d}}$ School of Mathematics and Physics, Queen's University Belfast (QUB), Belfast BT71 NN, UK

${ }^{\text {e }}$ Health Research Institute (HRI), University of Limerick (UL), Limerick V94 T9PX, Ireland f The Advanced Materials and Bioengineering Research (AMBER) centre

†These authors contributed equally to the work.

* Corresponding author: daniel.gamero@ua.es

* Corresponding author: micheal.scanlon@ul.ie 


\begin{abstract}
The versatility of conducting polymers (CPs) facilitates their use in energy conversion and storage, sensor, and biomedical technologies, once processed into thin films. Hydrophobic CPs, like poly(3,4-ethylenedioxythiophene) (PEDOT), typically require the use of surfactant additives, such as poly(styrenesulfonate) (PSS), to aid their aqueous processability as thin films. However, excess PSS diminishes CP electrochemical performance, biocompatibility, and device stability. Here, we report the electrosynthesis of PEDOT thin films at a polarised liquid|liquid interface, a method non-reliant on conductive solid substrates that produces free-standing, additive-free, biocompatible, easily transferrable, and scalable 2D PEDOT thin films of any shape or size in a single-step at ambient conditions. We demonstrate the PEDOT thin film's superior biocompatibility as scaffolds for cellular growth, opening immediate applications in organic electrochemical transistor (OECT) devices for monitoring cell behaviour over extended time periods, bioscaffolds and medical devices, without the requirement for physiologically unstable and poorly biocompatible PSS.
\end{abstract}

\title{
Main
}

Conducting polymers (CPs) find widespread applications in energy conversion and storage, sensor, optoelectronic, photovoltaic, bioelectronic and biomedical technologies. ${ }^{1-6}$ To satisfy this diverse set of applications, fabrication routes to CPs with desirable shapes/dimensions and compatibility with any substrate are required. The lightweight, flexible, and transparent nature of CPs makes them ideal for incorporation into technologies as thin films. ${ }^{7}$

Current methodologies to generate CP thin films have deficiencies that hinder technological progress. For example, multi-step film processing methods following chemical polymerisation require a complex of the $\mathrm{CP}$ and a hydrophilic surfactant, typically 
poly(styrenesulfonate) (PSS), to aid processability of technologically ubiquitous hydrophobic CPs, such as poly(3,4-ethylenedioxythiophene) (PEDOT). ${ }^{8}$ However, excess PSS diminishes CP conductivity, long-term stability, specific capacity, and biocompatibility (see Supplementary section 1.1 for more details). ${ }^{9-12}$ Electropolymerisation produces CP thin films in a single step, ${ }^{13}$ but is limited to deposition onto conducting surfaces and less easily adapted to the synthesis of composite materials. ${ }^{14}$ Vapour phase techniques provide another single-step method, capable of producing CP thin films with record-high electrical conductivity, ${ }^{15}$ but require high vacuum and/or temperatures, can be complicated or expensive to implement, and incompatible with heat-sensitive substrates. ${ }^{16}$

A liquid|liquid (L|L) interface provides a reproducible and defect-free environment to prepare and process free-standing two-dimensional (2D) thin films of nanomaterials, such as CPs, in a single step. ${ }^{17}$ By polarising certain $\mathrm{L} \mid \mathrm{L}$ interfaces, known as interfaces between two immiscible electrolyte solutions (ITIES), ${ }^{18-20}$ exquisite electrochemical control over the kinetics of interfacial electron transfer (IET) between oxidant and monomer redox couples in opposite phases is achieved. Progress to date in this field, interfacial electrosynthesis at an ITIES, is summarised in Supplementary section 1.2.

In this work, we report a major advance in the use of interfacial electrosynthesis at an ITIES to prepare free-standing, additive-free, reproducible, easily transferrable, scalable, and biocompatible PEDOT thin films in a single step at optimised ambient conditions. The external electrochemical driving force, the interfacial Galvani potential difference $\left(\Delta_{\mathrm{o}}^{\mathrm{w}} \phi\right)$, is applied using double potential step chronoamperometry (DPSCA). The IET reaction between an aqueous $\mathrm{Ce}^{4+}$ oxidant and organic EDOT monomer, and subsequent oligomer deposition at the $\mathrm{L} \mid \mathrm{L}$ interface, can be assisted or hindered by manipulation of $\Delta_{\mathrm{o}}^{\mathrm{w}} \phi$. We demonstrate an emergent enhanced biocompatibility of free-standing PEDOT thin films prepared at the ITIES, compared with films prepared by drop-casting commercial PEDOT:PSS surfactant- 
free ink, using normal human retina pigment epithelium (hTERT RPE-1) cells. The latter are a physiologically pertinent cell line given the application of PEDOT films in maculopathies..$^{21,22}$ We functionally modified the PEDOT and PEDOT:PSS films through the incorporation of bioactive proteins and monitored the consequential effects on cell behaviour in each case. These findings foreshadow potential applications as suitable 2D conductive substrates for RPE and electrically active photoreceptor cells and the development of organic electrochemical cell transistors (OECTs) capable of electrochemically monitoring cell growth over $>24 \mathrm{hr}$ periods.

\section{The mechanism of PEDOT interfacial electrosynthesis}

As illustrated in Fig. 1a(i)-(v), PEDOT thin film interfacial electrosynthesis progresses along five distinct stages with time. Initially, IET occurs between the aqueous $\mathrm{Ce}^{4+}$ oxidant and EDOT organic monomer forming monomeric radical cations $\left(\mathrm{EDOT}^{\bullet+}\right)$ in the diffusion layer on the organic side of the ITIES (Fig. 1a(i)). For IET to proceed with appreciable kinetics, the ITIES must be polarised positively (as discussed vide infra) with $\Delta_{\mathrm{o}}^{\mathrm{w}} \phi$ set to a value near the positive extreme of the Galvani polarisable potential window. EDOT $^{\bullet+}$ species are stabilised by the weakly coordinating organic anion, tetrakis(pentafluorophenyl)borate $\left(\mathrm{TB}^{-}\right),{ }^{23}$ and further couple with each other or EDOT monomers to form dimers in the diffusion layer. Continuous $\mathrm{EDOT}^{\bullet+}$ generation by IET and ensuing radical coupling steps ultimately lead to the formation of cationic PEDOT oligomers that also coordinate with $\mathrm{TB}^{-}$to maintain electroneutrality (Fig. 1a(i)). These coupling steps result in the release of protons on the organic side of the ITIES (Supplementary Fig. 1) that will be stabilised by the PEDOT thin film itself or by water present inside the film. ${ }^{24}$ 
(a)

(i)

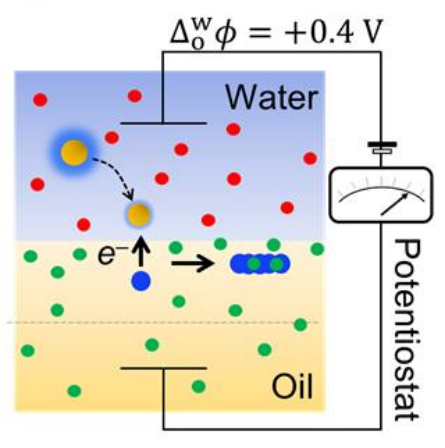

(ii)

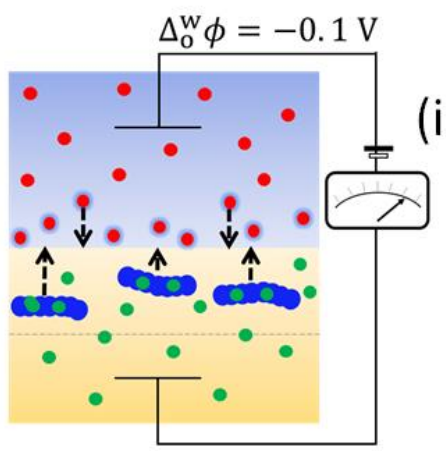

(iii)

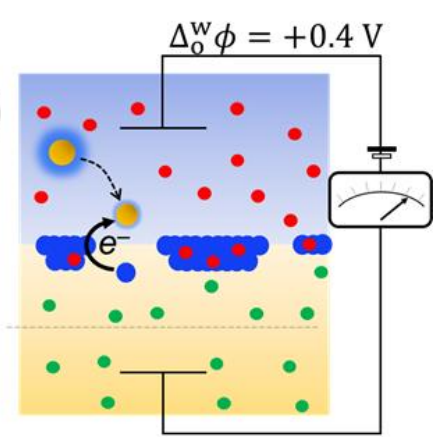

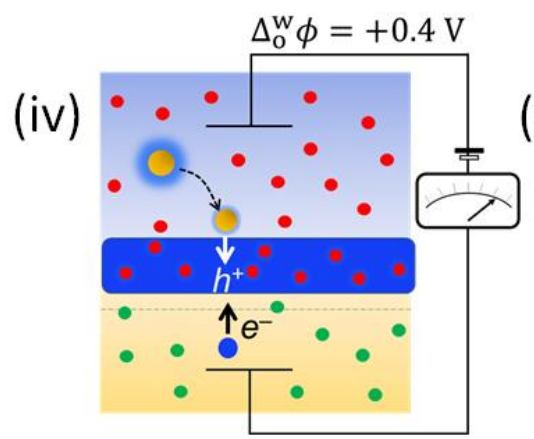

(b)

(i)

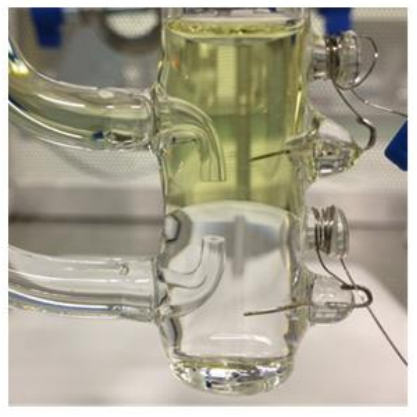

(ii) (v)

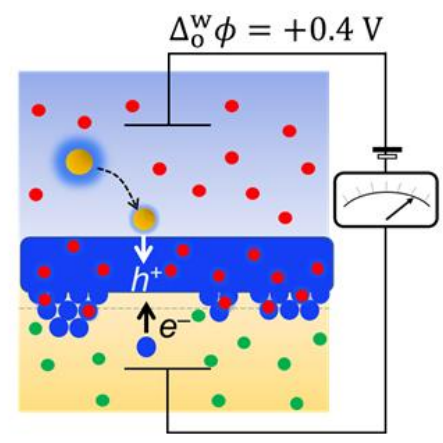

(c)
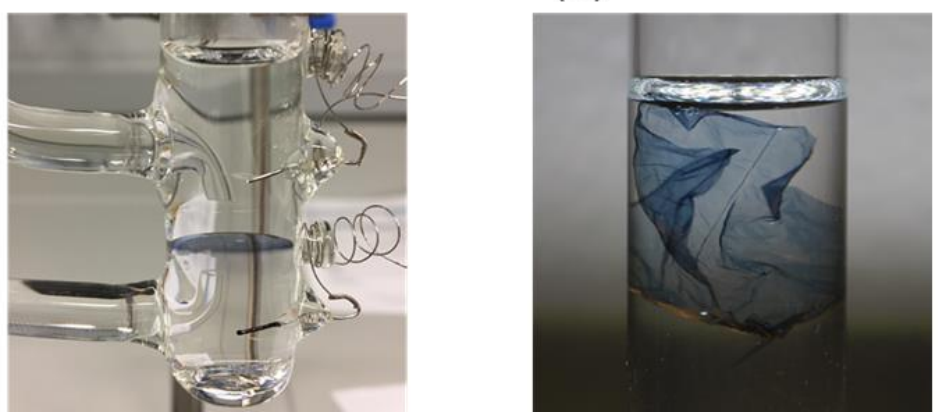

Fig. 1. The mechanism of PEDOT interfacial electrosynthesis at the interface between

two immiscible electrolyte solutions (ITIES). (a) The mechanism is schematically shown as five distinct steps with time: (i) interfacial electron transfer (IET) at a positive externally applied interfacial Galvani potential difference $\left(\Delta_{\mathrm{o}}^{\mathrm{w}} \phi=+0.4 \mathrm{~V}\right)$ between the aqueous $\mathrm{Ce}^{4+}$ oxidant and organic EDOT monomer to form cationic PEDOT oligomers, (ii) interfacial adsorption of the cationic PEDOT oligomers at a more negative $\Delta_{\mathrm{o}}^{\mathrm{w}} \phi(=-0.1 \mathrm{~V})$ through an ion-pairing interaction with the aqueous $\mathrm{SO}_{4}{ }^{2-}$ anions, (iii) autocatalytic IET between $\mathrm{Ce}^{4+}$ and EDOT at adsorbed PEDOT oligomer sites that act as interfacial bipolar electrodes, (iv) adsorbed PEDOT oligomer coalescence to form a highly compact 2D PEDOT thin film at the 
ITIES that is flat on both sides and heavily doped with aqueous $\mathrm{SO}_{4}{ }^{2-}$ anions, and (v) continued IET leading to a secondary growth process into the organic phase and the formation of a porous 3D structure on the organic-facing side as the thickness of the PEDOT thin film increases. (b) The four-electrode electrochemical cell (i) before and (ii) after interfacial electrosynthesis. The acidic aqueous phase, containing the yellow $\mathrm{Ce}^{4+}$ oxidant, is on top and the more dense $\alpha, \alpha, \alpha$-trifluorotoluene (TFT) organic solution containing the EDOT monomer is on the bottom. PEDOT forms exclusively as a thin blue film at the polarised liquid|liquid (L|L) interface. (c) A PEDOT thin film removed from a large ITIES and stored in an acetone/0.2 $\mathrm{M} \mathrm{H}_{2} \mathrm{SO}_{4}$ mixture to minimise gradual undoping.

Interfacial adsorption involving ion-pairing between the cationic PEDOT oligomers and aqueous electrolyte anions (herein $\mathrm{SO}_{4}{ }^{2-}$ ) takes place once the oligomers reach a critical size after an induction period (Fig. 1a(ii)). $\mathrm{SO}_{4}{ }^{2-}$ anions displace the weakly coordinating organic $\mathrm{TB}^{-}$anions during interfacial adsorption, ultimately becoming the sole dopant anion in the PEDOT thin film. This deposition process is driven by the energetically favourable reduction of the interfacial tension $(\gamma)$ between the two repulsive phases upon oligomer adsorption. ${ }^{25}$ Setting $\Delta_{\mathrm{o}}^{\mathrm{w}} \phi$ slightly negative of the potential of zero charge (PZC) is optimal for oligomer adsorption, ensuring the presence of a sufficient concentration of $\mathrm{SO}_{4}{ }^{2-}$ at the $\mathrm{L} \mid \mathrm{L}$ interface to participate in ion-pairing. Thus, as the IET and oligomer interfacial adsorption steps take place at different applied $\Delta_{o}^{\mathrm{w}} \phi$, potentiodynamic or multi-step potentiostatic are favoured over single-step potentiostatic electrochemical techniques. Also, a negative $\Delta_{\mathrm{o}}^{\mathrm{w}} \phi$ will facilitate the pumping of protons, generated on the organic side of the ITIES during the IET/radical coupling process at a positive $\Delta_{\mathrm{o}}^{\mathrm{w}} \phi$, to the aqueous phase via either direct ion transfer or the Grotthuss mechanism through the PEDOT thin film (Supplementary Fig. 2). 
In the next step, nucleation and growth of adsorbed PEDOT oligomers takes place at the interface. The PEDOT oligomers act as floating interfacial bipolar electrodes, providing abundant catalytic sites as electrical short-cuts to catalyse IET between the $\mathrm{Ce}^{4+}$ and EDOT species (Fig. 1a(iii)). ${ }^{26-28}$ Due to this autocatalytic effect, IET proceeds at a much lower overpotential than at a bare ITIES, with a higher kinetic rate. Thus, the PEDOT islands show rapid 2D growth, parallel to the $\mathrm{L} \mid \mathrm{L}$ interface. The gaps between individual rapidly growing islands of PEDOT disappear and a highly compact 2D PEDOT thin film coalesces at the ITIES that is flat on both sides, with a thickness of $\sim 50 \mathrm{~nm}$ (Fig. 1a(iv)). At this point, a physical barrier now exists between the $\mathrm{Ce}^{4+}$ and EDOT species at the ITIES. However, IET continues through the conductive PEDOT thin film and is subject to the influence of the diffusion of $\mathrm{SO}_{4}{ }^{2-}$ counter-anions through the film to maintain electroneutrality locally, i.e., "p-doping”. Continued IET initiates a secondary 3D growth process into the organic phase as the thickness of the PEDOT thin film increases. This controllable secondary growth process leads to the formation of a highly porous 3D structure, up to $\sim 850 \mathrm{~nm}$ thick (Fig. 1a(v)).

\section{Electrochemically initiating and controlling PEDOT thin film interfacial}

\section{electrosynthesis}

IET between $\mathrm{Ce}^{4+}$ and EDOT leading to a 2D PEDOT thin film is not a spontaneous process at an aqueous $\mid \alpha, \alpha, \alpha$-trifluorotoluene (TFT) interface (Supplementary Fig. 3). To initiate CP thin film formation, the aqueous|TFT interface must be polarised using a potentiostat in conjunction with a four-electrode electrochemical cell (Fig. 1b and Supplementary Fig. 4).

The resulting free-standing 2D PEDOT thin film could be recovered from the $\mathrm{L} \mid \mathrm{L}$ interface, washed with acetone and suspended in an acetone:sulfuric acid mixture (Fig. 1c) for storage before $e x$-situ characterisation or applications. Optimal conditions for thin film formation required a low concentration of oxidant (with the monomer always in excess), which is unconventional in chemical synthesis. A thermodynamic analysis of this biphasic system 
explains the need for an external electrochemical driving force to drive interfacial electrosynthesis with significant kinetics (Supplementary Information section 3).

Double potential step chronoamperometry (DPSCA) cycling provided the external driving force, with the four-electrode electrochemical cell configuration outlined in Fig. 2a. Current-time transients for the initial six DPSCA cycles are shown in Fig. 2b. A single DPSCA cycle involves first holding $\Delta_{\mathrm{o}}^{\mathrm{w}} \phi$ at $+0.4 \mathrm{~V}$ for $10 \mathrm{~s}$ to induce IET between $\mathrm{Ce}^{4+}$ and EDOT with appreciable kinetics, leading to a positive current-time transient and the formation of cationic PEDOT oligomers in the diffusion zone on the organic side of the ITIES. Next, $\Delta_{\mathrm{o}}^{\mathrm{w}} \phi$ is held at $-0.1 \mathrm{~V}$ for a further $10 \mathrm{~s}$ to induce PEDOT oligomer interfacial adsorption, leading to a negative current-time transient. The value of $-0.1 \mathrm{~V}$ was chosen as it is slightly negative of the PZC at a bare aqueous|TFT interface, determined as ca. $0 \mathrm{~V}$ by AC voltammetry (Fig. 2c), and optimal for oligomer adsorption as discussed vide supra. After 50 DPSCA cycles, a blue PEDOT thin film becomes visible at the aqueous|TFT interface (Fig. $1 b(i i))$. 

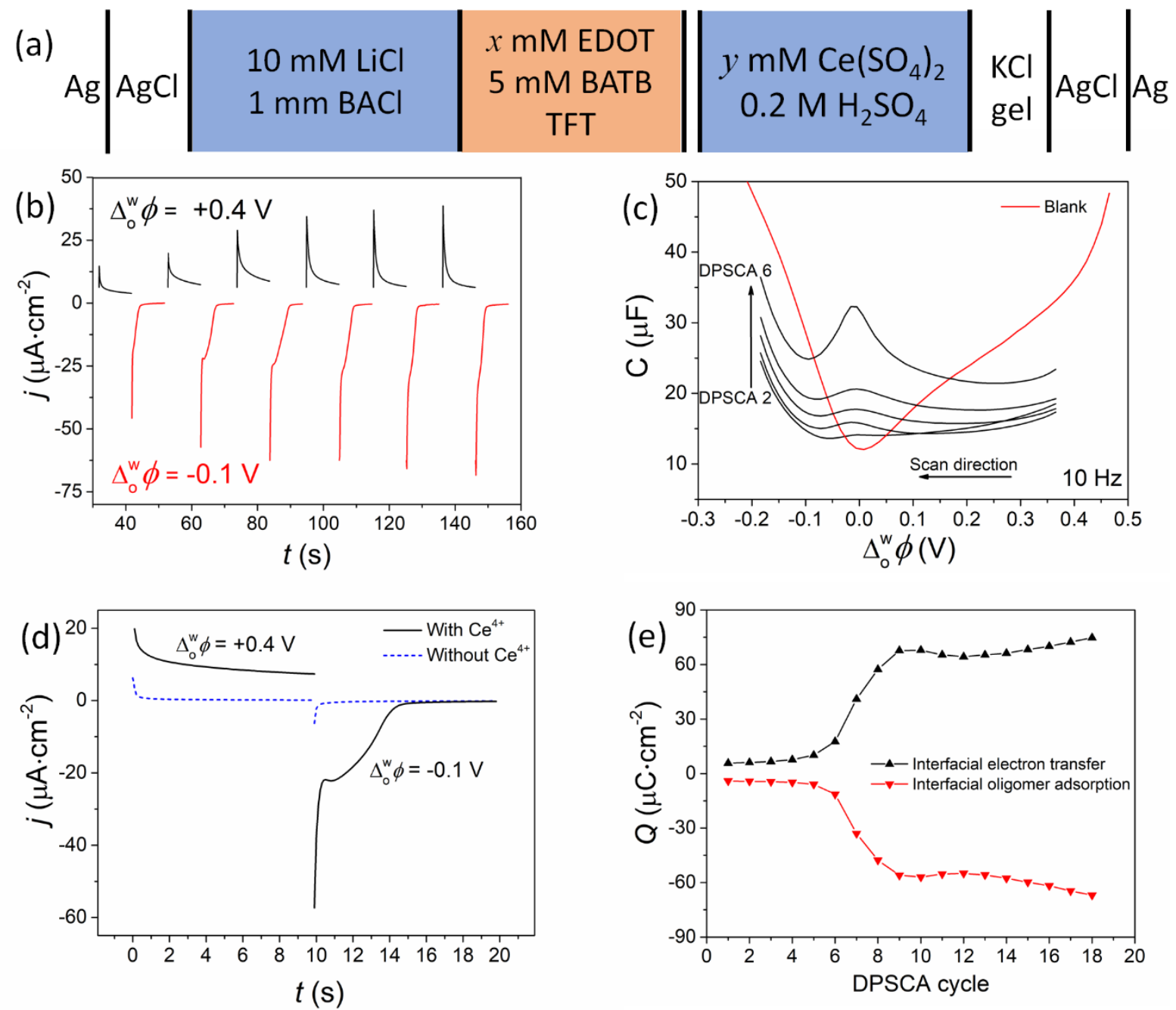

Fig. 2. Electrochemically initiating, controlling, and monitoring PEDOT thin film interfacial electrosynthesis. (a) Electrochemical cell configuration of the four-electrode electrochemical cell employed. For blank experiments $x$ and $y$ are both $0 \mathrm{mM}$. For interfacial electrosynthesis experiments $x$ and $y$ are 5 and $2 \mathrm{mM}$, respectively. In this four-electrode configuration, the organic $\mathrm{Pt}$ and $\mathrm{Ag} / \mathrm{AgCl}$ electrodes were connected to the counter and reference terminals, respectively, while the aqueous $\mathrm{Pt}$ and $\mathrm{Ag} / \mathrm{AgCl}$ electrodes were connected to the working and sensing terminals, respectively. All experiments were carried out under aerobic conditions. (b) Current-time transients recorded during double potential step chronoamperometry (DPSCA) cycles 5 to 10 in the presence of aqueous $\mathrm{Ce}^{4+}$ and organic EDOT. The first potential step was held at $\Delta_{o}^{w} \phi=+0.4 \mathrm{~V}$ for $10 \mathrm{~s}$, and the second at $\Delta_{o}^{w} \phi=-0.1 \mathrm{~V}$ for $10 \mathrm{~s}$. (c) Differential capacitance $(\mathrm{C} / \mu \mathrm{F})$ measurements performed at a 
bare aqueous|TFT interface (red line) and after DPSCA cycles 2 to 6 (black lines) in the presence of aqueous $\mathrm{Ce}^{4+}$ and organic EDOT. The frequency was $10 \mathrm{~Hz}$, the amplitude was $10 \mathrm{mV}$ and the scan direction was from positive to negative potential. (d) Control DPSCA experiments. Current-time transients recorded during a DPSCA cycle with (black lines) and without (dashed blue lines) the aqueous $\mathrm{Ce}^{4+}$ oxidation present during PEDOT interfacial electrosynthesis. (e) Plot of the charge $\left(Q / \mu \mathrm{C} \cdot \mathrm{cm}^{-2}\right)$ for each potential step recorded for the first 18 DPSCA cycles. All electrochemical experiments were performed using the cell configuration outlined in Fig. 2a under aerobic conditions.

No change in charge was observed after 100 DPSCA cycles during control experiments without $\mathrm{Ce}^{4+}$ present (Fig. 2d). Trends in the kinetics of interfacial electrosynthesis with DPSCA cycle number were determined by analysing the charge magnitudes recorded from both the positive and negative current transients during the first 18 DPSCA cycles (Fig. 2e). These trends corroborated the mechanism outlined in Fig. 1a, as detailed in Supplementary section 4.1. Evidence of interfacial ion-pairing between adsorbed PEDOT oligomers and $\mathrm{SO}_{4}{ }^{2-}$ anions during interfacial electrosynthesis is provided by comparison of differential capacitance measurements of the blank aqueous|TFT interface and after DPSCA cycles 2 to 6 in the presence of $\mathrm{Ce}^{4+}$ and EDOT (Fig. 2c and Supplementary section 4.2). In situ parallel-beam UV/vis absorbance measurements show the depletion of $\mathrm{Ce}^{4+}$ on the aqueous side of the L/L interface with continuous DPSCA cycling and PEDOT thin film formation at the ITIES after 300 DPSCA cycles (Supplementary Fig. 11). The Tyndall effect was used to explore the partition of small PEDOT oligomers from the organic to aqueous phase during interfacial electrosynthesis (Supplementary Fig. 12).

\section{Microscopic analysis}


Scanning electron microscopy (SEM) of a 2D PEDOT thin film prepared by DPSCA (150 cycles) revealed an asymmetric "Janus" morphology (Fig. 3a and Supplementary Fig. 13). One side is flat and featureless at the nanoscale, while the other shows a rough, porous 3D structure. The PEDOT thin films adhere to any solid substrate, with the thin film closely following the contours and taking the shape of the surface (Supplementary Figs. 14 and 15). The asymmetric nature of the PEDOT thin film leads to each side having distinct physical properties. For example, sessile drop measurements highlight significant differences in hydrophobicity, with water droplet contact angles of $89.5^{\circ}$ and $112.6^{\circ}$ measured on the flat and rough sides, respectively (Fig. 3b). Atomic force microscopy (AFM) images of the topography of a PEDOT thin film folded back on itself clearly highlight the differences in surface roughness of either side (Fig. 3c(i)) and revealed that the thickness of a thin film prepared by 50 DPSCA cycles is ca. $60 \mathrm{~nm}$ (Fig. 3c(ii)-(iii)). 
(a)
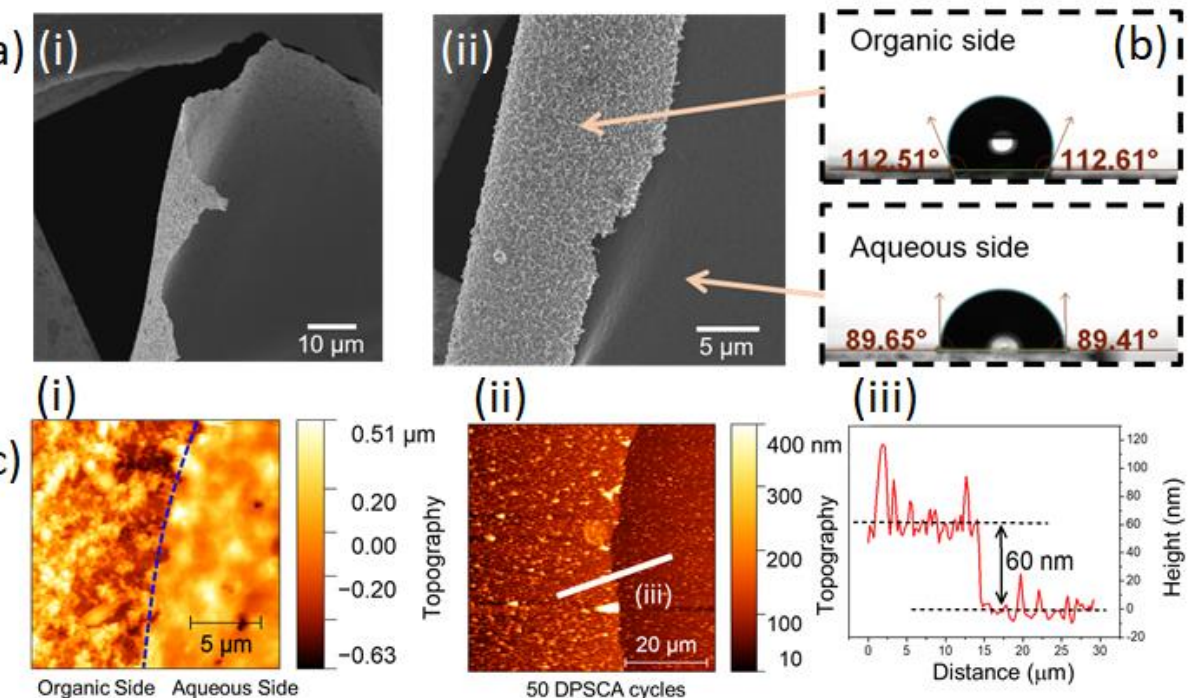

(ii)
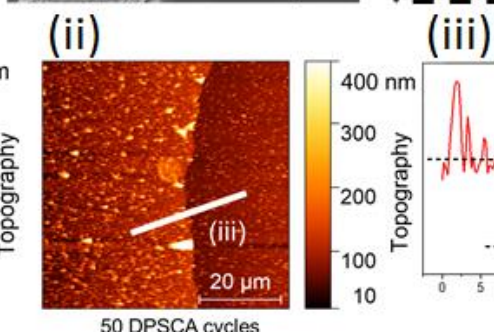

(iii)

(c)

(d)
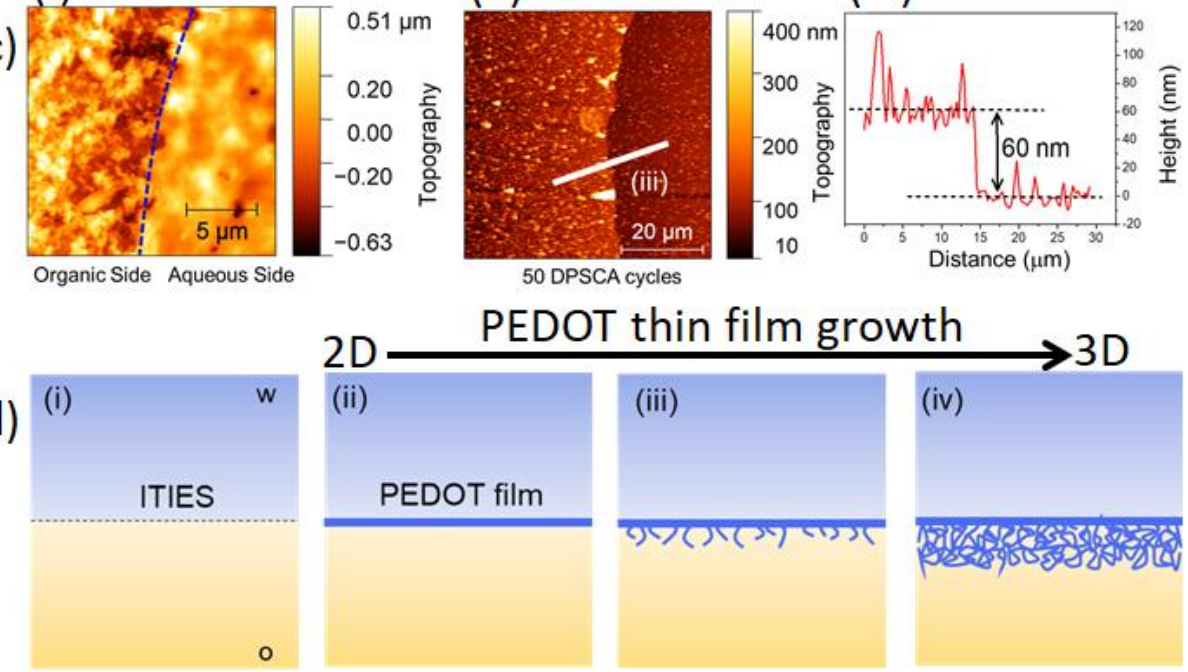

$3 \mathrm{D}$

(e)
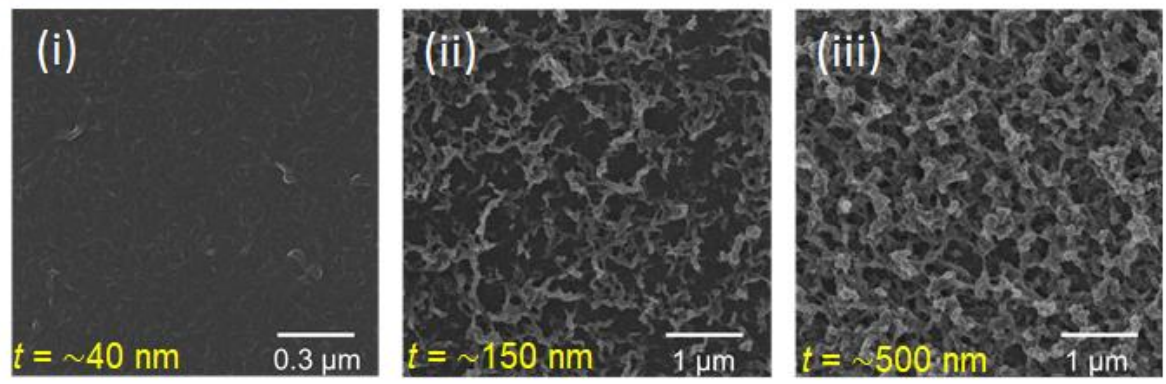

(f)
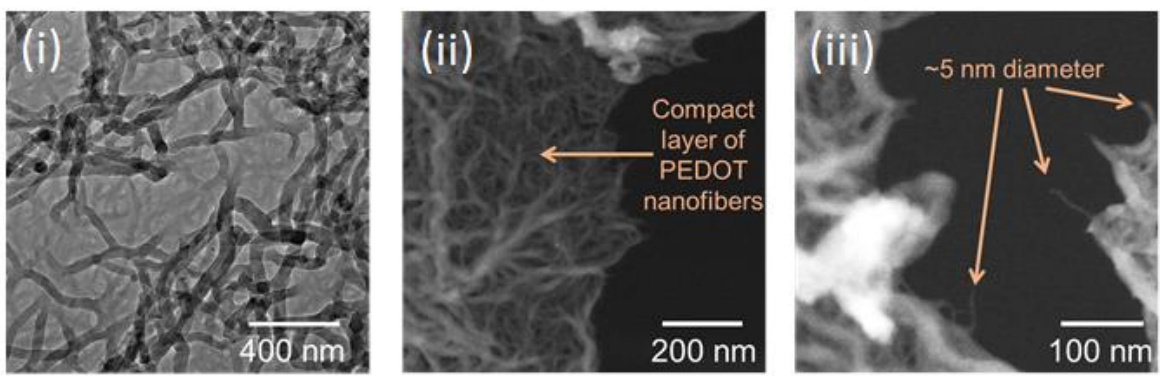

Fig. 3. Microscopic analysis of the PEDOT thin film. (a) Scanning electron microscopy

(SEM) images of a free-standing 2D PEDOT film electrosynthesised at the ITIES, showing the distinct morphologies of the "smooth" aqueous facing side and "rough" organic facing side. Additional SEM images are provided in Supplementary section 5.1. (b) Sessile drop contact angle measurements recorded on each side of the PEDOT thin film, highlighting the 
influence of the morphology on the measured hydrophobicity. (c) Atomic force microscopy (AFM) analysis of (i) the topography of a PEDOT thin film folded back on itself and (ii), (iii) the thickness of a PEDOT thin film on a flat gold substrate after 50 DPSCA cycles. (d) Schematic of the different stages of PEDOT thin film growth, from 2D to 3D, as a function of continued DPSCA cycling. (e) SEM images showing the organic facing side of the PEDOT thin film, demonstrating the controllable growth on the organic facing side of the thin film from $2 \mathrm{D}$ to $3 \mathrm{D}$ as a function of DPSCA cycling. The thickness $(t)$ of each thin film was determined by AFM (Supplementary Fig. 16). (f) (i) Bright field and (ii), (iii) dark field mode transmission electron microscopy (TEM) imaging shows that the film is a compact network of PEDOT nanofibers that have a diameter that ranges from $<5 \mathrm{~nm}$ up to $50 \mathrm{~nm}$. The arrows in (iii) point to PEDOT nanofibers with a diameter of ca. $5 \mathrm{~nm}$. All PEDOT thin films analysed were prepared by DPSCA cycling using the cell configuration outlined in Fig. 2a.

The evolution of the morphology of the organic facing side of the PEDOT thin film with DPSCA cycling is depicted schematically in Fig 3d(i)-(iv), with SEM images of each stage provided (Fig. 3e(i)-(iii)) and the thickness of each stage determined by AFM (Supplementary Fig. 16). Initially, after 50 DPSCA cycles, the thin film shows 2D growth parallel to the ITIES, a highly compact structure that is flat on both sides, and a thickness of $40-60 \mathrm{~nm}$ (Fig. 3e(i)). With continued interfacial electrosynthesis (up to 150 DPSCA cycles), secondary 3D growth begins to extend into the organic phase as the thickness of the PEDOT thin film increases (Fig. 3e(ii)). This controllable secondary growth process leads to the formation of a very porous 3D structure with a thickness of up to $\sim 850 \mathrm{~nm}$ after prolonged (> 300 DPSCA cycles) interfacial electrosynthesis (Fig. 3e(iii)).

Transmission electron microscopy (TEM) studies revealed that the PEDOT thin film is exceptionally stable under the TEM beam $(80 \mathrm{kV})$, signalling a high thermal conductivity 
and providing an opportunity to further investigate the PEDOT thin film's nanostructure. Both bright-field (Fig. 3f(i)) and dark-field (Fig. 3f(ii)-(iii)) mode TEM images show that the flat aqueous side consists of a compact layer of PEDOT nanofibers that run parallel to the ITIES. The diameter of the PEDOT nanofibers varies from $<5 \mathrm{~nm}$ to above $50 \mathrm{~nm}$. We propose that the nanofibers with a diameter of $<5 \mathrm{~nm}$ are first to be deposited at the ITIES during interfacial electrosynthesis, forming an initial compact layer. Subsequently, the nanofiber diameter increases as the thin film grows down into the organic phase.

\section{Spectroscopic, conductivity and electrochemical analysis}

Ex situ spectroscopic analysis was performed on PEDOT thin films transferred to suitable solid substrates (Supplementary section 6). A bipolaron band was observed by UV/vis absorbance, signifying that the PEDOT thin film is in an oxidised state ${ }^{29}$ and $p$-doped (Supplementary Fig. 17). Raman spectroscopy also confirmed that the PEDOT thin film is $p$ doped, with high $\pi-\pi$ conjugation and a benzenoid (coiled) configuration to the polymer chain (Supplementary Fig. 18), the more stable form when PEDOT is highly doped. ${ }^{30-32}$ An X-rayphotoelectron spectroscopy (XPS) survey spectrum showed the presence of only sulfur, carbon and oxygen (Supplementary Fig. 19), indicating that the cerium oxidant, boron and fluorine are not incorporated into the thin film. This implies that the organic $\mathrm{TB}^{-}$anion is not involved in $p$-doping and $\mathrm{SO}_{4}{ }^{2-}$ is the primary dopant. Further XPS analysis revealed that the doping level was estimated as $\sim 39 \%$ (Supplementary Fig. 20), in line with previous reports of the upper limits possible of PEDOT doping between $35-40 \%$. $^{33,34}$

Ex situ conductivity of the PEDOT thin film was determined as $554( \pm 77) \mathrm{S} \cdot \mathrm{cm}^{-1}$ (Supplementary Section 7.1), comparable to the highest conductivity value reported for a pristine PEDOT:ClO ${ }_{4}^{-}$film $\left(400-650 \mathrm{~S} \cdot \mathrm{cm}^{-1}\right)$ made by conventional electropolymerisation at a solid electrode surface in acetonitrile..$^{35,36}$ The in situ conductivity of a PEDOT thin film and a commercial PEDOT:PSS film drop cast and annealed directly onto a microelectrode 
array were compared in PBS buffer solution (Supplementary Fig. 22). The PEDOT and PEDOT:PSS films had maximum conductivities of $\sim 5.35 \mathrm{~S} \cdot \mathrm{cm}^{-1}$ and $\sim 1.2 \mathrm{~S} \cdot \mathrm{cm}^{-1}$, respectively, in their plateau regions, with the conductance window in the PEDOT thin film $0.4 \mathrm{~V}$ greater than that of the PEDOT:PSS film (Supplementary Fig. 23). The latter is advantageous for OECT devices working at lower potentials to avoid oxidative reactions or biological stress (if the active layer is to be functionalized with cells). Corresponding CVs of each film in PBS buffer solution were obtained using a range of scan rates and potential ranges (Supplementary Figs. 24 and 25). The PEDOT thin film remains doped over a very wide potential range (in comparison to the PEDOT:PSS film) and therefore has high conductivity even at negative potentials. At high scan rates, the PEDOT thin-film displays an ideal capacitive behaviour due to the polymer films high intrinsic conductivity and wide conductance window.

\section{Biocompatibility studies}

PEDOT:PSS films are being extensively explored for the treatment of visual impairment, particularly relating to amelioration or restoration of retinal function. ${ }^{21,22}$ However, the action of PSS severely hampers the biocompatibility and integrity of the resultant implantable biodevices (Supplementary section S8). In this context, the biocompatibility of PEDOT thin films electrosynthesised at the ITIES was compared with that of a drop cast PEDOT:PSS film using an adherent cell line derived from normal human retina pigment epithelium (hTERT RPE-1), see Fig. 4. 
(a)
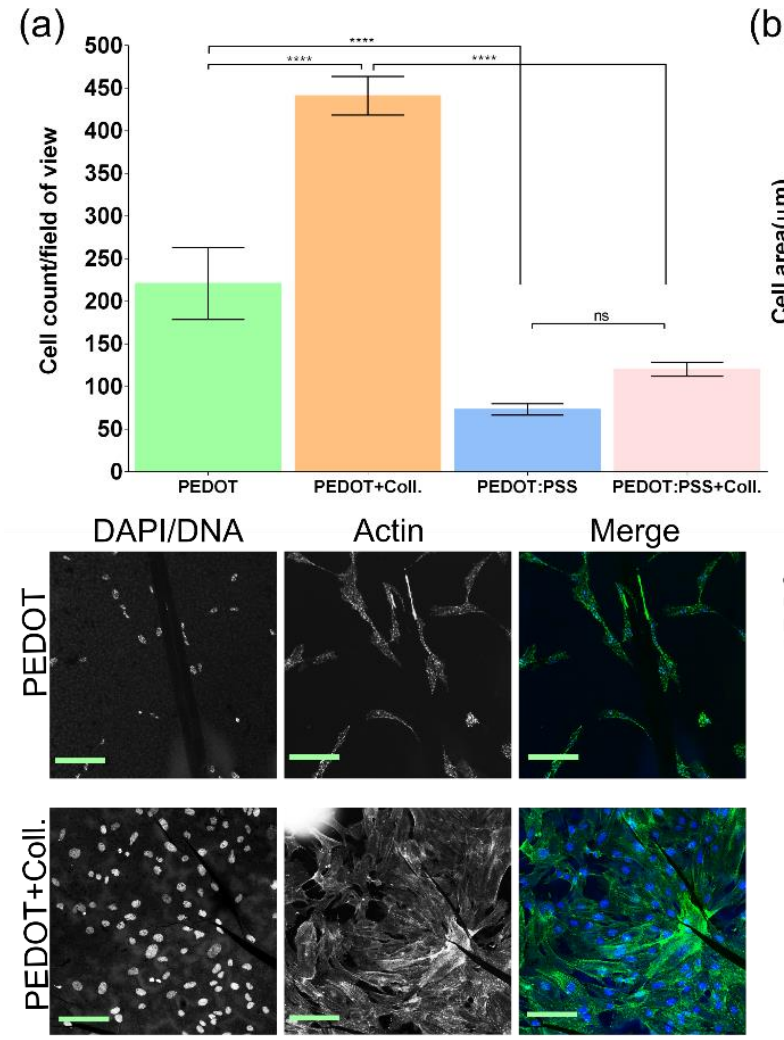

(b)
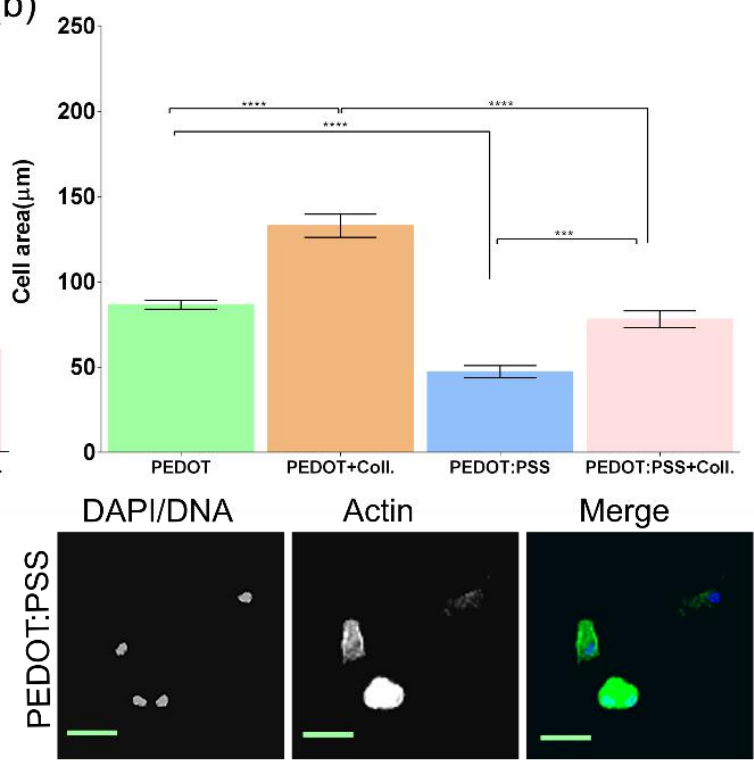

Actin
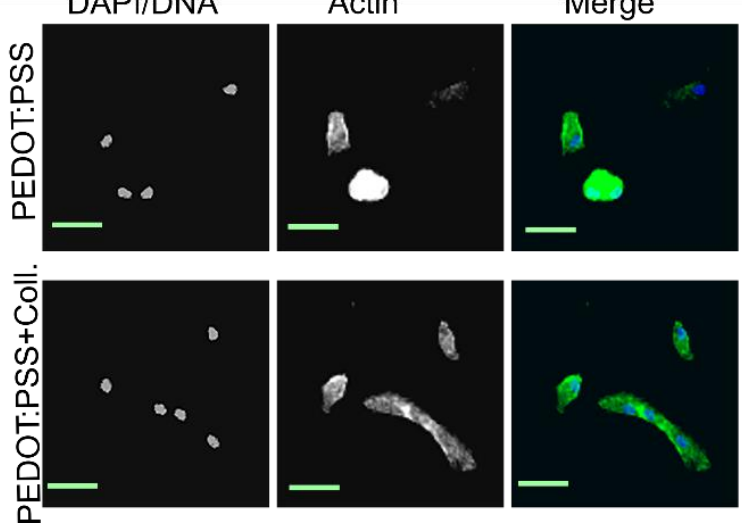

Fig. 4. Biocompatibility of PEDOT thin films prepared at the ITIES and drop-cast

PEDOT:PSS films with and without collagen functionalisation. (a) Cell proliferation

analysis (b) Cell area analysis for each sample film. Scale bar represents $50 \mu \mathrm{m}$.

Following 48 hours of cell growth on each film, marked differences were observed in hTERT RPE-1 cell growth dynamics (Fig. 4). Overall, in the presence of PEDOT thin films, cells exhibited a greater degree of proliferation and showed a stretched morphology, associated with actin bundle stress fibre formation, alluding to the more biocompatible nature of PEDOT versus PEDOT:PSS (Fig. 4a-b). These data re-enforce the challenges of using PSS at bio-interfaces and differentiate our investigations from many evaluating PEDOT:PSS in cell culture, where lack of obvious cytotoxicity is incorrectly construed as high biocompatibility. Next, each film was evaluated for suitability of active biomolecule incorporation. Collagen was selected as an active biomolecule due to its known influence on 
cell proliferation and adhesion, which could potentially mitigate the poor cellular growth seen on PEDOT:PSS samples. While only marginal effects were observed in cells grown on PEDOT:PSS films, functionalisation of PEDOT thin films resulted in a marked amplification in cell proliferation and cell spreading, indicative of robust adhesion receptor engagement and bioactivation of the cell cycle programme (Fig 4a).

\section{Discussion}

Interfacial electrosynthesis at an ITIES bridges the fields of purely homogeneous (chemical) electron transfer reactions between redox couples, which are difficult to control, and finely controlled (electrochemical) heterogeneous electron transfer at conventional solid electrodeelectrolyte interfaces. This work demonstrates that interfacial electrosynthesis directly produces PEDOT thin films of any shape or size in a single step, with distinctive molecular architectures inaccessible in bulk solution or at solid electrode-electrolyte interfaces, and emergent properties that facilitate technological advances. Electrochemical control of thinfilm nucleation and growth at the L|L interface allows fine control over the morphology, transitioning from 2D films (flat on both sides with a thickness $<50 \mathrm{~nm}$ ) to "Janus" 3D films (with a flat and rough side, each showing distinct physical properties, and thickness $>850$ $\mathrm{nm}$ ) by simply tuning the number of DPSCA cycles implemented. The PEDOT thin films were highly $p$-doped (approaching the theoretical limit), showed high $\pi-\pi$ conjugation, and were processed directly as thin films without insulating PSS. As a result, these films were highly conductive without post-processing, with their ex situ conductivity determined as 554 $( \pm 77) \mathrm{S} \cdot \mathrm{cm}^{-1}$ and a maximum in situ conductivity of $\sim 5.35 \mathrm{~S} \cdot \mathrm{cm}^{-1}$, more than 4 times greater than films prepared with the highest conductivity grade commercial PEDOT:PSS available from Sigma-Aldrich. Furthermore, when comparing the two conductive polymers, PEDOT thin films showed superior biocompatibility and suitability to be used as a scaffold for cellular growth without the need for further modification designed to promote cell adhesion 
or enhance viability. Bioactive molecules can be readily incorporated into PEDOT polymers as a further customisable parameter for cell growth studies. This opens an attractive avenue for potential new and improved OECT devices for monitoring cell behaviour over extended time periods, bio-scaffolds and medical devices, without the requirement for physiologically unstable and poorly biocompatible PSS.

Polarising the L $\mid \mathrm{L}$ interface provides an in-built ability to control the kinetics of IET between oxidant and monomer redox couples in opposite phases and probe the mechanism in situ electrochemically. These two features elevate this work over studies that involve spontaneous interfacial reactions (polycondensation, polyaddition, self-assembly), ${ }^{37-40}$ that permit only rudimentary chemical control over reaction kinetics and a limited ability to study the synthetic mechanism in situ. Our work unravels the underlying thermodynamic limitations and mechanism of PEDOT thin film interfacial electrosynthesis that progresses along five distinct stages with time. This underlying mechanism will be broadly generic for all aqueous oxidant/organic monomer combinations that are thermodynamically compatible to facilitate IET within the Galvani polarisable potential window (where the IET kinetics are under direct external electrochemical control). Such an understanding will allow us to identify suitable oxidant/monomer combinations, opening the field of interfacial electrosynthesis to other technologically critical CPs beyond PEDOT, e.g., poly(3hexylthiophene-2,5-diyl), commonly known as P3HT. The dimensions and geometry of freestanding thin films possible by interfacial electrosynthesis are only limited by the interfacial area and shape defined by the electrochemical cell. Furthermore, we demonstrate that interfacial electrosynthesis is a local and mild process that requires minimal reagents (e.g., low oxidant concentrations) and is thus highly compatible with large scale thin film production. In this regard, future work will explore polarising the L $\mid \mathrm{L}$ interface chemically, 
by establishing a suitable distribution potential through ion partition between the two phases, to scale-up thin film production.

\section{References}

1. Huang, J., Virji, S., Weiller, B. H. \& Kaner, R. B. Polyaniline nanofibers: Facile synthesis and chemical sensors. J. Am. Chem. Soc. 125, 314-315 (2003).

2. Xia, Y., Sun, K. \& Ouyang, J. Solution-processed metallic conducting polymer films as transparent electrode of optoelectronic devices. Adv. Mater. 24, 2436-2440 (2012).

3. Ghosh, S., Maiyalagan, T. \& Basu, R. N. Nanostructured conducting polymers for energy applications: Towards a sustainable platform. Nanoscale 8, 6921-6947 (2016).

4. Someya, T., Bao, Z. \& Malliaras, G. G. The rise of plastic bioelectronics. Nature 540, 379-385 (2016).

5. Zhang, Y., Ng, S. W., Lu, X. \& Zheng, Z. Solution-Processed Transparent Electrodes for Emerging Thin-Film Solar Cells. Chem. Rev. 120, 2049-2122 (2020).

6. Sultana, N., Chang, H. C., Jefferson, S. \& Daniels, D. E. Application of conductive poly(3,4-ethylenedioxythiophene):poly(styrenesulfonate) (PEDOT:PSS) polymers in potential biomedical engineering. Journal of Pharmaceutical Investigation 50, 437$444(2020)$.

7. D'Arcy, J. M. et al. Versatile solution for growing thin films of conducting polymers. Proc. Natl. Acad. Sci. U. S. A. 107, 19673-19678 (2010).

8. Yang, Y., Deng, H. \& Fu, Q. Recent progress on PEDOT:PSS based polymer blends and composites for flexible electronics and thermoelectric devices. Mater. Chem. Front. 4, 3130-3152 (2020).

9. Spencer, A. R. et al. Electroconductive Gelatin Methacryloyl-PEDOT:PSS Composite 
Hydrogels: Design, Synthesis, and Properties. ACS Biomater. Sci. Eng. 4, 1558-1567 (2018).

10. Cameron, J. \& Skabara, P. J. The damaging effects of the acidity in PEDOT:PSS on semiconductor device performance and solutions based on non-acidic alternatives. Mater. Horizons 7, 1759-1772 (2020).

11. Grossiord, N., Kroon, J. M., Andriessen, R. \& Blom, P. W. M. Degradation mechanisms in organic photovoltaic devices. Org. Electron. 13, 432-456 (2012).

12. Stöcker, T., Köhler, A. \& Moos, R. Why does the electrical conductivity in PEDOT:PSS decrease with PSS content? A study combining thermoelectric measurements with impedance spectroscopy. J. Polym. Sci. Part B Polym. Phys. 50, 976-983 (2012).

13. Zhou, M. \& Heinze, J. Electropolymerization of pyrrole and electrochemical study of polypyrrole: 1. Evidence for structural diversity of polypyrrole. Electrochim. Acta $\mathbf{4 4 ,}$ 1733-1748 (1999).

14. Reynolds, J. R., Thompson, B. C. \& Skotheim, T. A. Conjugated Polymers: Properties, Processing, and Applications. (CRC Press, 2019, 2019).

15. Wang, X. et al. High electrical conductivity and carrier mobility in oCVD PEDOT thin films by engineered crystallization and acid treatment. Sci. Adv. 4, eaat5780 (2018).

16. Evans, D. et al. Structure-directed growth of high conductivity PEDOT from liquidlike oxidant layers during vacuum vapor phase polymerization. J. Mater. Chem. 22, 14889-14895 (2012).

17. Zarbin, A. J. G. Liquid-liquid interfaces: A unique and advantageous environment to prepare and process thin films of complex materials. Mater. Horizons 8, 1409-1432 $(2021)$ 
18. Suárez-Herrera, M. F. \& Scanlon, M. D. On the non-ideal behaviour of polarised liquid-liquid interfaces. Electrochim. Acta 328, 1-9 (2019).

19. Gschwend, G. C., Olaya, A., Peljo, P. \& Girault, H. H. Structure and reactivity of the polarised liquid-liquid interface: what we know and what we do not. Curr. Opin. Electrochem. 19, 137-143 (2020).

20. Samec, Z. Electrochemistry at the interface between two immiscible electrolyte solutions (IUPAC Technical Report). Pure Appl. Chem. 76, 2147-2180 (2004).

21. Maya-Vetencourt, J. F. et al. A fully organic retinal prosthesis restores vision in a rat model of degenerative blindness. Nat. Mater. 16, 681-689 (2017).

22. Ferlauto, L. et al. Design and validation of a foldable and photovoltaic wide-field epiretinal prosthesis. Nat. Commun. 9, 1-15 (2018).

23. Kobayashi, H. Weakly coordinating bulky anions designed by efficient use of polyfluoro-substitution. J. Fluor. Chem. 105, 201-203 (2000).

24. He, S., Mukaida, M., Kirihara, K., Lyu, L. \& Wei, Q. Reversible protonic doping in poly(3,4-ethylenedioxythiophene). Polymers (Basel). 10, (2018).

25. Johans, C., Lahtinen, R., Kontturi, K. \& Schiffrin, D. J. Nucleation at liquid|liquid interfaces: Electrodeposition without electrodes. J. Electroanal. Chem. 488, 99-109 (2000).

26. Hotta, H., Akagi, N., Sugihara, T., Ichikawa, S. \& Osakai, T. Electron-conductor separating oil-water (ECSOW) system: A new strategy for characterizing electrontransfer processes at the oil/water interface. Electrochem. commun. 4, 472-477 (2002).

27. Hotta, H., Ichikawa, S., Sugihara, T. \& Osakai, T. Clarification of the Mechanism of Interfacial Electron-Transfer Reaction between Ferrocene and Hexacyanoferrate(III) 
by Digital Simulation of Cyclic Voltammograms. J. Phys. Chem. B 107, 9717-9725 (2003).

28. Gamero-Quijano, A., Molina-Osorio, A. F., Peljo, P. \& Scanlon, M. D. Closed bipolar electrochemistry in a four-electrode configuration. Phys. Chem. Chem. Phys. 21, 9627-9640 (2019).

29. Massonnet, N. et al. Improvement of the Seebeck coefficient of PEDOT:PSS by chemical reduction combined with a novel method for its transfer using free-standing thin films. J. Mater. Chem. C 2, 1278-1283 (2014).

30. Garreau, S., Duvail, J. L. \& Louarn, G. Spectroelectrochemical studies of poly(3,4ethylenedioxythiophene) in aqueous medium. Synth. Met. 125, 325-329 (2002).

31. Łapkowski, M. \& Proń, A. Electrochemical oxidation of poly(3,4ethylenedioxythiophene) - 'in situ' conductivity and spectroscopic investigations Synth. Met. 110, 79-83 (2000).

32. Garreau, S., Louarn, G., Buisson, J. P., Froyer, G. \& Lefrant, S. In situ spectroelectrochemical Raman studies of poly(3,4-ethylenedioxythiophene) (PEDT). Macromolecules 32, 6807-6812 (1999).

33. Bubnova, O. et al. Optimization of the thermoelectric figure of merit in the conducting polymer poly(3,4-ethylenedioxythiophene). Nat. Mater. 10, 429-433 (2011).

34. Shi, W., Zhao, T., Xi, J., Wang, D. \& Shuai, Z. Unravelling Doping Effects on PEDOT at the Molecular Level: From Geometry to Thermoelectric Transport Properties. J. Am. Chem. Soc. 137, 12929-12938 (2015).

35. Zotti, G., Zecchin, S., Schiavon, G. \& Groenendaal, L. B. Conductive and magnetic properties of 3,4-dimethoxy- and 3,4-ethylenedioxy-capped polypyrrole and polythiophene. Chem. Mater. 12, 2996-3005 (2000). 
36. Zotti, G. et al. Electrochemical and XPS studies toward the role of monomeric and polymeric sulfonate counterions in the synthesis, composition, and properties of poly(3,4-ethylenedioxythiophene). Macromolecules 36, 3337-3344 (2003).

37. Piradashvili, K., Alexandrino, E. M., Wurm, F. R. \& Landfester, K. Reactions and polymerizations at the liquid-liquid interface. Chem. Rev. 116, 2141-2169 (2016).

38. Booth, S. G. \& Dryfe, R. A. W. Assembly of Nanoscale Objects at the Liquid/Liquid Interface. J. Phys. Chem. C 119, 23295-23309 (2015).

39. Scanlon, M. D., Smirnov, E., Stockmann, T. J. \& Peljo, P. Gold Nanofilms at LiquidLiquid Interfaces: An Emerging Platform for Redox Electrocatalysis, Nanoplasmonic Sensors, and Electrovariable Optics. Chem. Rev. 118, 3722-3751 (2018).

40. Poltorak, L., Gamero-Quijano, A., Herzog, G. \& Walcarius, A. Decorating soft electrified interfaces: From molecular assemblies to nano-objects. Appl. Mater. Today 9, 533-550 (2017).

\section{Methods}

Materials. All chemicals were used as received without further purification. All aqueous solutions were prepared with ultra-pure water (Millipore Milli-Q, specific resistivity 18.2 $\mathrm{M} \Omega \cdot \mathrm{cm}$ ). The organic solvent $\alpha, \alpha, \alpha$-trifluorotoluene (TFT, 99+\%) was received from Acros Organics. The organic monomer 3,4-ethylenedioxythiophene (EDOT, 97\%), cerium (IV) sulfate $\left(\mathrm{Ce}\left(\mathrm{SO}_{4}\right)_{2}, 99 \%\right)$, sulfuric acid $\left(\mathrm{H}_{2} \mathrm{SO}_{4}, 95.0 \%\right)$, lithium chloride ( $\left.\mathrm{LiCl}, \geq 99 \%\right)$ and tetraethylammonium chloride (TEACl, 99\%) were purchased from Sigma-Aldrich. Lithium tetrakis(pentafluorophenyl)borate diethyletherate (LiTB) was received from Boulder Scientific Company. The organic electrolyte salt bis(triphenylphosphoranylidene)ammonium tetrakis(pentafluorophenyl)borate (BATB) was prepared by metathesis of equimolar solutions 
of $\mathrm{BACl}$ and $\mathrm{LiTB}$ in a methanol-water $(2: 1 \mathrm{v} / \mathrm{v})$ mixture. The resulting precipitates were filtered, washed and recrystallised from acetone. For comparative studies, a PEDOT:PSS ink (1.1\% in $\mathrm{H}_{2} \mathrm{O}$, surfactant-free, high-conductivity grade) was purchased from Sigma-Aldrich. For biocompatibility studies, Dulbecco's Modified Eagle's Medium (DMEM), 10\% fetal bovine serum (FBS), 1\% penicillin/streptomycin, sodium bicarbonate (S8761), collagen in phosphate buffer saline (PBS) solution (125-50) Trypsin-EDTA solution (T4049), anti-actin FITCE conjugated dye (19083) and 4',6-diamidino-2-phenylindole (DAPI) were obtained from Sigma-Aldrich. Also, $1 \%$ GlutaMAX (35050061) was obtained from ThermoFisher Scientific, Zombie green (423111) from Biolegend and normal human Retina Pigment Epithelium (hTERT RPE-1; CRL-4000) from ATCC.

Interfacial electrosynthesis at a polarised aqueous|organic interface. Interfacial electrosynthesis was carried out at an ITIES formed between an acidic aqueous solution, containing $0.2 \mathrm{M} \mathrm{H}_{2} \mathrm{SO}_{4}$ electrolyte and $2 \mathrm{mM} \mathrm{Ce}\left(\mathrm{SO}_{4}\right)_{2}$ as the oxidant, and an organic TFT solution, containing $5 \mathrm{mM}$ BATB electrolyte and $5 \mathrm{mM}$ EDOT as the monomer (see Fig. 2a). The interfacial Galvani potential difference at the polarised aqueous|TFT interface $\left(\Delta_{\mathrm{o}}^{\mathrm{w}} \phi\right)$ was controlled externally using an Autolab PGSTAT204 from Metrohm (Netherlands), in conjunction with NOVA software version 2.1.2. and a specialised four-electrode electrochemical cell. A detailed schematic of the latter is shown in Supplementary Fig. 4 and images are provided in Fig. 1b. Two reference electrodes positioned on different sides of the aqueous|TFT interface measured the potential difference across the interface. Platinum counter electrodes placed in each phase allowed the flow of electric current. The geometrical surface area of the aqueous|TFT interface was $\sim 1.66 \mathrm{~cm}^{2}$. To enhance the diffusion of the $\mathrm{Ce}^{4+}$ oxidant in the aqueous phase, a homemade stirrer was positioned approx. $2 \mathrm{~cm}$ above the water|TFT interface. A glass capillary with a U-bend was used as the stirrer, which was attached to an electric motor that operated at $240 \mathrm{rpm}$. A theoretical background to such 
experiments at an ITIES can be found in several articles and book chapters. ${ }^{18-20,41,42}$ All electrochemical measurements were carried out in ambient aerobic conditions at room temperature (RT, $\left.21^{\circ} \mathrm{C}\right)$. The methodology to calibrate all electrochemical data obtained at the ITIES to the Galvani potential scale is described in Supplementary section 2.

Interfacial electrosynthesis was initiated using a double potential step chronoamperometry (DPSCA) method. The first potential step was held at $\Delta_{\mathrm{o}}^{\mathrm{w}} \phi=+0.4 \mathrm{~V}$ for $10 \mathrm{~s}$. The second potential step was then held at $\Delta_{\mathrm{o}}^{\mathrm{w}} \phi=-0.1 \mathrm{~V}$ for $10 \mathrm{~s}$. This double potential step was repeated up to 300 times, depending on the desired thickness of the PEDOT thin film.

AC voltammetry was performed after each of the first five DPSCA cycles. as shown in Fig. 2c. Differential capacitance was calculated from the interfacial admittance recorded using an Autolab FRA32M module in combination with the Autolab PGSTAT204 at a frequency of $10 \mathrm{~Hz}$ and root mean square (RMS) amplitude of $10 \mathrm{mV}$. The scan direction was from negative towards more positive potentials, from ca. -0.20 to $+0.35 \mathrm{~V}$.

\section{Electropolymerisation using a closed bipolar electrochemical cell (CBPEC) in a 4-}

electrode configuration. The bipolar electrode consisted of two individual Au disc electrodes ( $\mathrm{CH}$ instruments, USA), one in each compartment acting as the aqueous pole $\left(\mathrm{P}_{\mathrm{w}}\right)$ or organic $\left(\mathrm{P}_{\mathrm{o}}\right)$, that were connected with an electric wire. Each compartment contained a $\mathrm{Pt}$ wire driving electrode and a reference electrode. The aqueous reference electrode was a $\mathrm{Ag} / \mathrm{AgCl}(\mathrm{KCl}$ gel) electrode, while the organic reference electrode was a $\mathrm{Ag} / \mathrm{AgCl}$ wire immersed in an "organic reference solution" that established a liquid junction with the organic solvent. The organic reference solution was an aqueous solution of $1 \mathrm{mM} \mathrm{LiCl}$ and $10 \mathrm{mM} \mathrm{BACl}$. The measured potential was calibrated onto the Galvani potential scale using the procedure described above and elsewhere. ${ }^{43}$ Before use, two individual pole Au disc electrodes were polished and cleaned electrochemically in $0.2 \mathrm{M} \mathrm{H}_{2} \mathrm{SO}_{4}$. The organic pole 
was also rinsed with de-ionised water, acetone and TFT solvent prior to CBPEC electrochemistry. Experiments were performed in ambient conditions. The concept of a CPBEC experiment with immiscible aqueous|organic electrolyte solutions is shown schematically in Supplementary Fig. 7 and the CPBEC configuration used for the electropolymerisation of organic EDOT by aqueous $\mathrm{Ce}^{4+}$ is described in Supplementary Scheme 1.

Microscopic analysis. $\underline{\text { Scanning electron microscopy (SEM) and transmission electron }}$ microscopy (TEM) samples were prepared by drop casting PEDOT thin films onto $\mathrm{Cu}$ mesh grids, as shown by SEM images in Supplementary Figs. 14 and Supplementary Fig. 15. SEM measurements were performed with a Thermo Scientific ${ }^{\mathrm{TM}}$ Helios $^{\mathrm{TM}}$ G4 CX DualBeam ${ }^{\mathrm{TM}}$ microscope at $5 \mathrm{kV}$ using a Through-the-Lens Detector (TLD) in immersion mode. TEM measurements were performed at $80 \mathrm{kV}$ with a ThermoFisher USA (formerly FEI Co.) Titan Themis Z (60-300 kV) TEM equipped with a double Cs (spherical aberration) corrector, a high brightness electron gun (xFEG), an electron beam monochromator, and a Gatan Quantum 966 imaging filter. Atomic force microscopy (AFM) was undertaken in tapping mode using a Pt-coated Si tip (PPP-EFM, Nanosensors, Switzerland) with a stiffness constant of $2.8 \mathrm{~N} \cdot \mathrm{m}^{-1}$ and resonance frequency of $67 \mathrm{kHz}$ on an MFP-3D infinity AFM system (Asylum Research, USA).

Spectroscopic analysis. PEDOT thin films were recovered from the aqueous|TFT interface, washed, and then drop cast onto an ITO glass slide for UV/vis-NIR absorbance analysis, a stainless-steel specimen disc for Raman analysis and a polished silicon wafer substrate for Xray-photoelectron spectroscopy (XPS) analysis. Raman spectroscopic measurements were performed using a LabRAM HR Evolution Raman Confocal Microscope (Horiba, France) with LabSpec 6 software. Measurements were performed with a $783 \mathrm{~nm}$ excitation line laser source. Calibration was performed with a Silicon standard $\left(520.07 \mathrm{~cm}^{-1}\right) . \underline{\mathrm{UV} / \mathrm{vis}-\mathrm{NIR}}$ 
absorbance measurements were made using a DH-2000-BAL deuterium-halogen light source and a Maya 2000 Pro Spectrometer. XPS measurements were performed using a Kratos ULTRA spectrometer (Kratos Analytical, UK) with monochromatic Al Ka radiation energy of $1486.58 \mathrm{eV} . \mathrm{C} 1 \mathrm{~s}$ at $284.8 \mathrm{eV}$ was used as the charge reference to determine the core level binding energies.

In situ UV/vis absorbance measurements were performed using the parallel beam configuration that is illustrated in Supplementary Fig. 10. The light source, DH-2000-BAL deuterium-halogen (Ocean Optics), was directed through the electrochemical cell at an angle parallel to the aqueous|TFT interface, with the use of an UVFS coated plano convex lens (Thorlabs) and an iris diaphragm (Thorlabs). After transmission through the electrochemical cell, the light source was focused by a second UVFS coated plano convex lens (Thorlabs) to a Maya 2000 Pro Spectrometer (Ocean Optics).

Sessile drop water contact angle measurements. Contact angle measurements were made on the opposite sides of the PEDOT thin films using a Theta Attension Optical Tensiometer from Biolin Scientific (Sweden) controlled using OneAttension software version 2.0. Water contact angle measurements were performed with Milli-Q deionized water $(18.2 \mathrm{M} \Omega \cdot \mathrm{cm})$ at RT $\left(21^{\circ} \mathrm{C}\right)$. A droplet volume of $10 \mu \mathrm{L}$ was used.

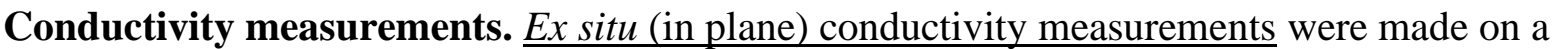
four-strip conductivity electrode (ALS, Japan, see optical images in Supplementary Fig. 21) using the method described elsewhere. ${ }^{44-46}$ The four-strip method was favoured over the conventional four-point-probe setup due to the better contact achieved with the former as the probe tips caused significant tearing of the PEDOT thin film sample. The current-voltage (I$V)$ relationship of the PEDOT thin film was measured with an Autolab PGSTAT204 potentiostat from Metrohm (Netherlands), controlled using NOVA software version 2.1.2. Ex situ conductivity measurements were performed at RT $\left(21^{\circ} \mathrm{C}\right)$ in ambient conditions. 
$\underline{\text { In situ conductivity measurements }}$ were performed in phosphate buffer saline (PBS; $137 \mathrm{mM} \mathrm{NaCl}, 2.7 \mathrm{mM} \mathrm{KCl}$ and $10 \mathrm{mM}$ phosphate buffer solution, $\mathrm{pH}$ 7.4) solution using an interdigitated microelectrode array (NanoSPR, USA) operated in a transistor-like configuration, as illustrated in Supplementary Fig. 22, with a Pt wire counter electrode and $\mathrm{Ag} / \mathrm{AgCl}(\mathrm{KCl}$ gel) reference electrode. The microelectrode array was operated with a WaveDriver 20 bipotentiostat from Pine research (USA) controlled by AfterMath software version 1.6. PEDOT thin films prepared by interfacial electrosynthesis were drop cast onto the interdigitated microelectrode array, and oven dried at $60^{\circ} \mathrm{C}$ for $2 \mathrm{~h}$. For the PEDOT:PSS films, a quantity of the PEDOT:PSS ink was drop cast onto the electrode surface and dried for $2 \mathrm{~h}$ in the oven at $60^{\circ} \mathrm{C}$. The thickness of the PEDOT films were determined by AFM prior to in situ conductivity measurement. In situ conductivity measurements were performed in a nitrogen environment. In situ measurements were started at positive potential, where both PEDOT films were fully oxidised, and swept to negative potential.

Three-electrode electrochemical characterisation. Electrochemical characterisation of PEDOT films was performed in PBS solution by cyclic voltammetry (CV) using a threeelectrode configuration. The working electrode was either a Au disc electrode or an indium tin oxide (ITO) glass slide $(60 \Omega)$. The working electrode was coated by the PEDOT film sample as described for the in situ conductivity experiments for PEDOT thin films prepared by interfacial electrosynthesis and the PEDOT:PSS films. Immobilised PEDOT films were rinsed with DI water and PBS solution before use. The counter and reference electrodes were a Pt wire and a $\mathrm{Ag} / \mathrm{AgCl}(\mathrm{KCl}$ gel) electrode, respectively. The Au electrode was used to investigate the electrochemical doping/undoping of the PEDOT films due to the Au electrodes wide potential window. Electrochemical doping/undoping of the PEDOT films was performed in anaerobic conditions (Supplementary Fig. 24). ITO electrodes were used to 
investigate the PEDOT films scan rate dependence (Supplementary Fig. 25) Scan rate studies were performed in aerobic conditions.

Biocompatibility studies. For control experiments, PEDOT:PSS films were prepared by drop-casting a volume of PEDOT:PSS ink (1.1\% in $\mathrm{H}_{2} \mathrm{O}$, surfactant-free, high-conductivity grade) onto cell culture treated plastic wear. The PEDOT:PSS film was dried overnight in oven at $60^{\circ} \mathrm{C}$.

PEDOT thin films prepared by interfacial electrosynthesis were allowed to attach to the surface of a cell culture treated plastic wear and washed 3 times with $1 \times$ Phosphate buffer saline (PBS) to remove any residual species. Films were sterilized with $30 \mathrm{~min}$ of ultraviolet light (UV) exposure. Following sterilization, a group of samples were coated with $10 \mu \mathrm{g} / \mathrm{mL}$ collagen in PBS solution for 2 hours at $37^{\circ} \mathrm{C}$. Residual collagen solution was removed.

An adherent cell line of normal human Retina Pigment Epithelium (hTERT RPE-1) of passage number $<10$ was used for this study. Prior to seeding on the PEDOT films, hTERT RPE- 1 cells were grown under normal cell culture conditions at $37{ }^{\circ} \mathrm{C}$ and $5 \% \mathrm{CO}_{2}$ in Dulbecco's Modified Eagle's Medium (DMEM) supplemented with 10\% fetal bovine serum (FBS), $1 \%$ penicillin/streptomycin, $1 \%$ GlutaMAX, and sodium bicarbonate until they reached $80 \%$ confluency. Afterwards, cells were trypsinised, counted, and diluted to a concentration of 5,000 cells $/ \mathrm{mL}$. Cells were maintained in culture for 48 hours then stained with fixable dead cell labelling dye following manufacturer's instructions (Zombie green) and fixed with 4\% paraformaldehyde solution (PFA) / 4\% sucrose/ PBS for 15 min at RT, washed with $1 \times$ PBS with $0.2 \%$ Triton-X for 10 min at RT, and blocked with $10 \%$ FBS $/ 1 \times$ PBS (blocking solution (BS)) for 1h at RT. Afterward, cells were incubated with anti-actin FITCE conjugated dye diluted in BS for $1 \mathrm{hr}$ at RT. After $3 \times 5$ min washes with $1 \times \mathrm{PBS}$, the cells were counterstained with 4',6-diamidino-2-phenylindole (DAPI). 
For the biocompatibility study, samples were imaged on the ImageXpress Micro Confocal High-Content Imaging System (Molecular Devices) with a 10X S Plan Apo objective. 9 fields of view per sample in each wavelength were analysed using open source CellProfiler software (v 4.0.5). ${ }^{47}$ Briefly, image sets corresponding to various fluorescent channels in each field of view were subjected to normalization and analysis pipelines where cell object segmentation was undertaken using DAPI nuclear identification. Mean cell areas were quantified using ImageJ, by the bounding pixel area associated with segmented objects denoted through actin labelling.

Statistical analysis was performed using Graph Pad Prism Version 8.4.2 (464) (GraphPad Software, La Jolla, CA, USA), and values tested for significance using one-way ANOVA followed by Tukey post-hoc tests, or t-tests for pair-wise comparison. Statistical tests were two tailed with a significance level of $\alpha \leq 0.05$. Significances are stated with $p$ values $<0.05 * ;<0.01 * * ;<0.001 * * *$. Results are shown as standard error of means.

\section{Data availability}

All data is available from the corresponding author M.D.S on request.

\section{References}

41. Samec, Z. Dynamic electrochemistry at the interface between two immiscible electrolytes. Electrochim. Acta 84, 21-28 (2012).

42. Peljo, P. \& Girault, H. H. Liquid/Liquid Interfaces, Electrochemistry at. in Encyclopedia of Analytical Chemistry (John Wiley \& Sons, Ltd, 2012). doi:10.1002/9780470027318.a5306.pub2

43. Gamero-Quijano, A., Herzog, G. \& Scanlon, M. D. Bioelectrochemistry of Cytochrome $\mathrm{c}$ in a closed bipolar electrochemical cell. Electrochem. commun. 109, 106600 (2019). 
44. Cho, B. et al. Single-crystal poly(3,4-ethylenedioxythiophene) nanowires with ultrahigh conductivity. Nano Lett. 14, 3321-3327 (2014).

45. Seng, S., Shinpei, T., Yoshihiko, I. \& Masakazu, K. Development of a handmade conductivity measurement device for a thin-film semiconductor and its application to polypyrrole. J. Chem. Educ. 91, 1971-1975 (2014).

46. Guo, Z. et al. Modulating the electrical conductivity of metal-organic framework films with intercalated guest $\pi$-systems. J. Mater. Chem. C 4, 894-899 (2016).

47. McQuin, C. et al. CellProfiler 3.0: Next-generation image processing for biology. PLoS Biol. 16, 1-17 (2018).

\section{Acknowledgements}

M.D.S. acknowledges Science Foundation Ireland (SFI) under Grant no. 13/SIRG/2137 and the European Research Council through a Starting Grant (Agreement no. 716792). A.G.-Q. and A.H. acknowledge funding received from Irish Research Council (IRC) Government of Ireland Postdoctoral Fellowship Awards (Grant Numbers GOIPD/2018/252 and GOIPD/2020/821, respectively). R.L. and S.M acknowledge funding received from IRC Government of Ireland Postgraduate scholarships (Grant Numbers GOIPG/2018/2132 and GOIPG/2019/3693).

\section{Author contributions}

R.A.L and A.G.-Q. co-carried out and co-designed the initial proof-of-concept interfacial electrosynthesis experiments yielding PEDOT thin films at the ITIES and co-wrote the first manuscript draft. For this article, R.A.L. co-wrote the manuscript, carried out and coanalysed all interfacial electrosynthesis experiments at the ITIES in a 4-electrode configuration, all closed bipolar electrochemical cell (CPBEC) experiments, all electrochemical experiments with PEDOT film modified solid electrodes in a 3-electrode 
configuration, all UV/vis-NIR and Raman experiments and all in situ and ex situ conductivity experiments. R.A.L. also prepared PEDOT thin films for subsequent microscopic and spectroscopic analysis. A.G.-Q. co-wrote the manuscript, supervised and co-designed all of R.A.L's experiments and developed the methodologies of the CPBEC and in situ/ex situ conductivity experiments. A.G.-Q. also carried out the sessile drop water contact angle measurements and prepared PEDOT thin films for the biocompatibility studies. S.M. carried out and K.M. supervised the biocompatibility study using the hTERT RPE-1 cell line, with and without collagen incorporated. S.M. and K.M. also co-wrote the manuscript. M.C. carried out and U.B supervised the collection of the TEM images shown in Fig. 3f. A.H. obtained all scanning electron microscopy (SEM) images. F.L. carried out the X-ray photoelectron spectroscopy (XPS) experiments and analysed the data. A.K. carried out the atomic force microscopy (AFM) experiments and analysed the data. M.D.S. co-wrote the manuscript, obtained the funding, conceived, and supervised the project.

\section{Competing interests}

The authors declare no competing interests. 